\title{
Library Literacy Program: Library as Battleground for Fighting Fakenews
}

\author{
William Taala ${ }^{1}$, Filoteo B. Franco Jr. ${ }^{2}$, Parisa H. Sta. Teresa ${ }^{1}$ \\ ${ }^{1}$ Fakeeh College for Medical Sciences, Jeddah, KSA \\ ${ }^{2}$ Prince Mohammad Bin Salman College for Business and Entrepreneurship, Jeddah, KSA \\ Email: wtaala@fakeeh.care,wtaala@fcms.edu.sa,ffranco@mbsc.edu.sa,phteresa@fakeeh.care
}

How to cite this paper: Taala, W., Franco Jr., F.B. and Teresa, P.H.S. (2019) Library Literacy Program: Library as Battleground for Fighting Fakenews. Open Access Library Journal, 6: e5296.

https://doi.org/10.4236/oalib.1105296

Received: February 25, 2019

Accepted: March 15, 2019

Published: March 18, 2019

Copyright () 2019 by author(s) and Open Access Library Inc.

This work is licensed under the Creative Commons Attribution International License (CC BY 4.0).

http://creativecommons.org/licenses/by/4.0/

\section{Open Access}

\begin{abstract}
Library literacy programs have continued to play a pivotal role in ensuring that students only consume the information necessary to their studies in the wake of fake news. Before the introduction of the Internet as an information tool, fake news was dealt with amicably before reaching out to numerous persons. Nevertheless, the advent of the Internet has contributed to the spread of fake news to unparalleled levels. Educational institutions of higher learning should ensure that their literacy programs meet the required threshold and equip the learners with prerequisite skills to differentiate between fake news and factual academic content as well as promote a reading culture and critical thinking skills. The research paper also highlights the medical student situation in Saudi Arabia that has been transformed from five institutions to approximately 21 institutions illustrating the need for library literacy skills to students in the quest to improve the quality of medical studies in the country. Cognizant of the fake journals accessed at the click of a button with unlimited access, the need for library literacy programs is timely. The social, economic and political development of a country highly depends on the educational competence of its population. For this reason, countries across the world have continued to allocate a substantial amount of money towards educational development by opening up new academic facilities as well as enhancing teacher population.
\end{abstract}

\section{Subject Areas \\ Information Science}

\section{Keywords}

Fake News, Library Literacy Program, Saudi Arabia 


\section{Introduction}

In the wake of technological avenues that act as information repositories, information literacy has been overtaken by fake news that has proliferated information outlets; from the mainstream media, alternative media to academic libraries. To arrest this situation, there is an emphatic need for institutions of higher learning to prioritize information literacy programs aimed at empowering students and faculty members with the prerequisite information based on their fields of study through proper identification, selection, interpretation, and usage of the data available on library shelves as well as credible academic sites. In institutions of higher learning, the quest for information ends at the library where students and faculty members alike turn to electronic and physical material. To this effect, libraries are neatly stocked with the latest literature not only to promote scholarly excellence.

However, the lack of structured library literacy programs in most documentation stores negatively affects the output of research cognizant of the proliferation of fake news outlets that ultimately find space in libraries. In light of this, there is a need for learning institutions to conduct library literacy programs in the pursuit of scholarly materials for students in the research process. In essence, the proliferation of fake news notably in the wake of web-based research awakened by Internet connectivity has withered the scope of research in learning facilities emphasizing the need for literacy programs to equip learners with specialized skills to differentiate between fake content and genuine and credible literature in the learning process.

This research essay advocates persistent library research programs will soon transform the quality for research in an institution of higher learning as it seeks to empower the information literacy levels of students through a selection of valuable data from a pool of alternatives.

\section{Library Literacy}

Upon entry to an institution of higher learning, the primary goal of a student is to excel and progress to professional career growth. This is energized through structured resolve to engage in research activities. However, the attainment of career goals bred in academic institutions faces threats associated with amplification of fake news in schools thus the need for information literacy programs. Librarians, for this reason, play a critical role in molding a student's performance aware that teachers spend a fraction of their time on students. This negatively affects the academic growth of students mindful of the vulnerability of the students to consume fake news projected as academic realities (Baro, Endouware, \& Ubogu, 2011) [1].

The world today is home to numerous information sources ranging from media outlets, social media sites as well as opinion leaders in society such as religious leaders, political leaders, and scholars. However, these information sources generate information from multiple unverified and verified sources. This in mind, 
there is a need for individuals notably students to be extensively informed on the best available information sources in the quest to make informed decisions ultimately ease problem-solving in the society. For example, religious leaders play a critical role in nurturing human behavior through among others doctrines. This, nevertheless, affects the thought patterns of faithful who make conclusions based on these ideologies. In academia, for this reason, scholars shape the intellectual thoughts of students. On the other hand, the critical role of these information shapers is gradually facing a threat from information communication technologies cognizant of the proliferation of fake news outlets (Baro, Endouware, \& Ubogu, 2011) [1].

Librarians, in this regard, help students identify the material commensurate to their academic disciplines (Qureshi, Qureshi, \& Qureshi, 2018) [2]. Excellent performance in schools is not a reflection of a person's ability to locate the best content in an array of alternatives. For example, with the influx of the Internet in learning institutions, students are unlikely to differentiate between the best information for consumption primarily because of informational illiteracy.

Aware of the proliferation of data in a library, selection of the much-needed information is a problem student experience in the course of their studies thus the need to understand library literacy. Upon entry into a library, the librarian's critical role is to guide the library user concerning information search in the databases available. This is primarily because library users are not sufficiently informed on the best practices in the library aimed at boosting their research output. For instance, a learner may result in the use of outdated literature because of lack of library literacy thus the need to undertake library literacy programs.

Information literacy, a component of library literacy, refers to a situation whereby a person has access to information but identifies the information he/she requires as well as come up with strategies on how to use it effectively. The library is home to numerous information covering diverse academic and non-academic disciplines thus the need to learn how to use the best information in the presence of fake news/data all over. In the information age era, the proliferation of information across various platforms has devalued the real essence of information in decision making as well as research purposes. With a click of a button, a learner using an online platform has numerous data streams with identical information thus the need to sieve the best against the unneeded information (Okuonghae \& Ogiamien, 2016) [3].

Additionally, this illustrates the need for information literacy in library services to understand, choose and use the best and credible information. In essence, library literacy helps users to select the best in accordance to their needs thus improving their research outcome. In medical studies, for example, lack of library literacy skills is vulnerable to the improper articulation of medical issues (Zervas, Stavrou, \& Kounoudes, 2015) [4].

\subsection{Fake News}

In the information age era, sources of information have increased drastically in 
line with an unprecedented increase in Internet connectivity coupled with ease of access to technological devices. Unlike in the past when individuals were inclined to single sources of information such as television and print media, a multiplicity of information sources in modern day society has created ample space for the proliferation of fake news.

Understandably, information dispatch in the past was censored, and only the information relevant for public consumption was released. For example, lack of social media in the past compelled the population to result to broadcast and print media' structured content. The advent of the Internet opened up the propagation of fake news on a diverse scale. This is because the Internet heralded new dawn to the dissemination of news from anonymous sources. The Internet, the principal tool of information broadcasting, was preferred primarily because of reach effectiveness and immediacy. For example, a single tweet from the United President Donald Trump can reach out to numerous persons within a click of a button eliciting massive reactions across the globe. This illustrates the impact that fake news may have on academia notably when propagated from anonymous sources. For instance, when a medical student picks up information on the Internet and acknowledges it as the reality and factual representation of a concept primarily because it is repeated extensively, this amounts to consumption of fake news in academia (Courtney, 2018) [5].

The proliferation of news outlets in modern day society away from the conventional professionally profound trained journalism to yellow journalism has transformed access, dissemination, and understanding of news content. Mindful of escalating web-based content in the wake of the digital library, drawing the line between excellent and credible content to the sensational and advertorial content that finds away in news programming is gradually becoming a hectic experience notably in the wake of decreased information literacy among consumers of news content.

In a learning environment, it is important to scale up the analysis of fake news in the wake of opinionated content presumed to be a factual representation of issues. For example, a nutrition student who spends considerably long hours watching television content by nutritional analysts may be easily swayed by opinions, and lack of information literacy skills compromise his/her reasons and reflection of factuality.

In the era of the Internet sharing of information on online platforms is at unprecedented levels questioning the validity and accuracy of the information. For example, the young generation has embraced technology on social media platforms sharing information extensively leaving negligible space for verification of the factuality of the data, a situation that degenerates to spread of fake news.

Introduction of the Internet was projected to ease the transmission of information across geographical borders as well as simplify the social-economic interests of the masses. However, its entry in the education sector has gradually tilted its benefits southwards. This in mind, the Internet has become a critical 
information search hub for students, therefore, promoting the proliferation of fake news online. For example, instead of searching for credible academic journals to seek answers to a medical problem by medical students, ease of access of Internet-enabled technological devices such as laptops, and smart phones have negatively affected the quality of research becoming insurmountable threats to the sustainability of academic decorum. The anonymous features associated with Internet sources of information extensively promote fake news. This implies that a person in the comfort of his or her study room generates resources without visiting the library (Nichols, 2017) [6].

\subsection{Medical Education in Saudi Arabia}

The medical education situation in Saudi Arabia has experienced a great transformation with medical colleges increasing from 5 medical schools to 21 medical schools in approximately ten years. This illustrates the emphatic need for critical resources to assist students, and faculty members generate great research. Initially, the Saudi medical schools had a teacher-centered approach to studies limiting access to valuable information for the students cognizant of the limited teacher-student ratio (Bin Abdulrahman, Harden, \& Patrício, 2012) [7].

Nevertheless, a drive for sustainable and innovative medical education programs for students in Saudi Arabia has changed the curriculum to be more centered on resource allocation to libraries such that students get ample opportunities to supplement already acquired data from the teacher. This has, however, not been successful notably because of the proliferation of secondary information avenues including and not limited to social media and other Internet-based research tools.

Interestingly, this has affected the research outcome of medical students jeopardizing the healthcare sector. An increase in fake news predicts a deem future for medical students, but stringent library literacy programs are inevitable whereby students are advised and guided on the best research materials in the literacy programs in an attempt to spice up research outcomes.

Alsuraihi, Almaqati, Abughanim, and Jastaniah (2016) [8] argue that social media is gradually becoming an important communication tool in the $21^{\text {st }}$ century penetrating academia at uncontrollable levels. This is because the social media platforms act as alternative information focal points thus students use in the compilation of their research projects. However, access to the factual information continues to affect the productivity of research projects undertaken by medical students. The consistency of social media information makes it popular thus eludes the factual aspect of it. For example, a tweet or post that is shared extensively on a social media platform may be inclined to be true and factual. It is important to acknowledge that not all truthful information is factual (Alsuraihi et al., 2016) [8].

Bin Abdulrahman et al. (2012) [7] stress that the learning environment that students are exposed to contribute greatly to their success in class and ultimately 
during their career growth. In light of this, there is a need for the learning environment to come up with library literacy programs to empower students with authentic literature in their studies. To sustain local and international students, medical education in Saudi is taught using the English language. This is primarily to incorporate the diversity concerning culture, language, race, and nationalities of students. With an increasing number of medical colleges in Saudi, quantity, as opposed to quality, will be an eventual crisis.

\section{Ways in Which Library Literacy Programs Help Fight Fake News among Medical Students}

Decoding messages and literature varies from one person to another. To this end, interpretation of data notably in school is heavily reliant to a person's culture, religion, age, and gender. In light of this, penetration of fake news in the learning institutions is not only aligned towards mass media but also encrypted cultural ideologies that persons use in making decisions.

For this reason, incorporation of these forms of fake news is injurious to the realization of academic success notably for medical students. Ideally, medical students heavily rely on scientific research to come up with solutions thus integration of fake news only serves to dilute the scientific aspect of medical science. For example, before establishing that a person is suffering from a particular disease, emphatic diagnosis is conducted for easier treatment and management of ailments thus usage of fake news to compromise the diagnostic procedure only serves to limit critical thinking for students.

Forging partnerships besides is another way that libraries literacy programs help in the reduction of fake news among medical students. This implies that library literacy programs are structured such that they use diverse platforms including and not limited to digital platforms to encourage students to seek knowledge on digital platforms such as web applications. Through web-based programs, institutions of higher learning are likely to reach out to numerous students in colleges, unlike physical orientation programs that are limited to space and resources. In the digital era, adoption of cloud services has helped students link up with library content irrespective of the resources therein.

\subsection{Digital Libraries}

In the wake of information communication technologies across the globe, learning institutions are gradually resulting in infrastructure development projects to accommodate the rising need for digital libraries. In terms of medical students, therefore, library literacy programs help in the fight against fake news through the establishment and recognition of digital libraries as alternative avenues for knowledge-based learning. Physical libraries, for example, are not properly structured to accommodate a wide range of students' because of among others limited physical space and literature. In this regard, encouraging students to use digital libraries compared to the unverified sources amplifies their quest 
for knowledge as well as align their needs towards uptake of digital resources (Mandalia \& Parekh, 2017) [9].

Medical sciences students need for current, and evidence-based data in their problem-based learning and clinical problems reveals the important role of library literacy programs in the studies. Since print databases are limited to serve an ever-increasing student population, schools are gradually adopting electronic learning platforms to sustain the population as well as create room for expansion of data storage.

Electronic databases have multiple research materials ranging from journal articles, e-books to conference papers. For this reason, it is important for universities to expand their libraries to incorporate emerging trends such as and not limited to virtual libraries to increase access. However, the lack of skills required in the identification and usage of data has led to decreased research output. In light of this, library literacy skills empower students in making informed data choices (Mandalia \& Parekh, 2017) [9].

\subsection{Information Retrieval Orientation and Discovery Learning}

Upon completion of high school studies, students are upbeat of the smooth institution of higher learning studies. However, upon entry into the colleges, they are bombarded with information cognizant of the multiplicity of information sources. To this end, there is an emphatic need for libraries to conduct literacy programs for learners irrespective of their academic disciplines through among others conducting information retrieval orientation programs. In their initial stages of learning, medical students through properly structured information literacy programs get an opportunity to engage in discovery learning interestingly acclimatizing themselves with the available research skills.

Through the information retrieval orientation programs, librarians help the students use the physical and virtual catalog in the quest to highlight the necessary information in their research work. In the absence of a librarian's help, it is difficult for users to identify, retrieve and purposely use the information contained in the catalogs.

Fundamentally, the information found at the library is vulnerable to fake news cognizant of lack of sufficient analysis. This in mind, it is important to acknowledge the role of librarians in guiding users on the ethical, critical and general use of the information repository. Aware of the critical challenges that face students transitioning from high school to universities, library literacy is inevitable.

Medicine, unlike other fields of study, requires timely, factual and credible information cognizant of its impact on the society and repercussions associated with negligence and substandard data. With the introduction of the Internet in most institutions of higher learning, libraries are on the verge of collapse with fake news taking considerable space in academia (Storie \& Campbell, 2012) [10]. Medical students are programmed to undertake information literacy skills in an 
attempt to improve their research findings and critical thinking abilities.

In light of this, awareness in the use of library resources plays a critical role mindful of the fact that patronage at the facility without prior knowledge of its use is equitable to time mismanagement. Precisely, when a student knows where to locate, identify and use information placed in the library, he/she spends less time in the library's catalog thus improving his/her research outcome.

\subsection{Empowerment}

Multiple access points for information has contributed to information overload with negligible practices out in place to herald new dawn where library users become empowered with the vital information in the face of multiplicity. For this reason, library literacy programs have fought fake news among students through partnership programs with media institutions and social media developers to tighten their information dispatch mechanisms. For example, in the recent past, WhatsApp among other social networking companies have come up with policies to ensure the reduction of fake news among members of the public as well as students in learning institutions through the limitation of forwarded messages.

Fake news, in essence, distorts the critical thinking ability of an individual when they are repeated numerous times with limited forwards and sharing of information, a new practice aimed at reducing the negative impact in information literacy. By empowering information consumers' notably medical students always to seek verification of information from reputable sources prior to interpreting information as the factual representation of data. This is through reputable journals. For example, a medical student must not be in a hurry to grasp information for use in the studies from unrecognized outlets such as social media sites.

\subsection{Access to Credible Materials}

In the wake of counterfeit products finding a way into the market, a similar scenario is replicated in the library section with fake literature finding space on library shelves. In this regard, library literacy programs help fights fake news proliferation through the placement of library materials that are authentic and meet the desired international standards. Introduction of digital libraries, for example, helps reduce the proliferation of fake literature in the library since such literature may compromise the role of libraries in information provision.

Through the library literacy programs, librarians and active participants in the educations sector conduct routine checks on the material placed in libraries and weed out fake news publication. For instance, media organizations offer material to be used in libraries in the form of newspapers; however, lack of sufficient analysis, this strategy of equipping libraries may lead to an increase in fake news as students turn to newspapers for information.

Additionally, library literacy programs record great results in the presence of 
adequate infrastructure in terms of available literature and recruitment of professional librarians among others. In essence, the availability of multiple resources at the library from diverse fields of study actively works towards the fight against the use of fake news by students in their academic research projects. For example, if a student is able to easily locate academic literature using the library's proper documentation infrastructure as exemplified by the Saudi Digital Library, he/she reduces the impact of fake news in the studies. Precisely, the Saudi Digital Library partnership with professional publishers across the world helps in reducing the penetration of the fake news in the learning institutions as the available data on the online platform is strategically aligned towards academic prosperity (Stein-Smith, 2017) [11].

With multiple publishers flooding the education market armed with large portions of data, there is a need to ensure that the usable information meets the prerequisite threshold for academic integrity. In this regard, library literacy programs help students to identify the correct choices of information from an array of alternatives. In essence, online databases are easily available to users, and the stringent analysis of literature uploaded therein helps in transforming the literacy levels of students. For example, librarians capitalize on such online databases to shield students away from the vagaries of poor publishing outlets. Ideally, it is important to acknowledge that the multiplicity of documents in a library does not translate to the inclusion of factual data thus the need for verification procedures.

\section{The Extent of Fake News Concentration among Medical Students in Saudi Arabia}

Social media has penetrated learning institutions becoming key agents in the spread of fake news in the world. From websites to web applications, social media has transformed the concept of good research with students using social media reports as a true representation of factual research. As a result, this has changed the education landscape in the country. Statistics in a study done in Saudi Arabia revealed that 74.4 percent of students used social media in their medical institution as an educational tool (Alsuraihi et al., 2016) [8]. In light of this, it is evident that the inclusion of social media as an education tool in medical school was an enabling factor in the promotion of fake news in the studies. For example, cognizant of the vulnerability of social media in the learning process and the proliferation of non-factual information, penetration of it creates an avenue for fake news.

Fake news has affected education to the extent of scientific journals in the medical field of study. Mindful of the important role that journals play in the achievement of academic goals, the use of fake news in journals has negatively influenced academic growth in Saudi Arabia and beyond. According to Tin et al. (2014) [12], the quest to have research material published for academic purposes has exploited the increasing open access publishing. 
In this regard, the published material lacks the authenticity required as publishers accept multiple manuscripts but lack the desire to undertake the prerequisite avenues to ensure conformity to set academic standards. As a result, fake news gets the opportunity to trickle down to students whose allegiance to journal articles is compromised. For example, due to lack of sufficient library literacy programs to assist in the identification and use of the authentic journals in the information repositories, students are easily swayed by the easy availability of the journals associated with fake news thus affecting the academic standards of their papers (Agosto, 2018) [13].

In essence, a student may write a research paper full of correct citation procedures, but the journals used in the compilation of the information therein fail to meet the academic standards primarily because of heavy usage of fake news in compiling the articles. This illustrates the emphatic need for library literacy skills to be incorporated in learning institutions as a precursor towards academic integrity. Fake publishers in the academic sphere have negatively contributed to the escalation of fake news in the medical schools (Tin et al., 2014) [12].

With an increased appetite for academic excellence, the past decade has recorded significant growth in the number of academic journals penetrating the market for use by students and faculty members. However, the emerging demand for academic literature has ideally compromised the production of factual and credible resources for use. To this end, the high number of journals mushrooming from the publication agencies has flooded the market with fabricated content mimicking the peer-reviewed scientific journals.

In this regard, medical students in Saudi are vulnerable to these fake journals out to propagate fake news as a true representation of reality ultimately compromising academic quality. For example, when a student uses a journal that is not factual, he/she is likely to compromise education quality and subsequently tarnish professional reputation. This underscores the importance of library literacy programs in academic institutions to help students identify fake journals (Neely-Sardon \& Tignor, 2018) [14].

Open-access journals have opened room for the proliferation of fake news across institutions of higher learning extensively downplaying the important role of library literacy programs in empowering students on the best journals. Fundamentally, unrestricted access to journals further complicates the credibility equation. For example, if a student has open access to academic material without limitation, he/she is likely to undermine the library's role in academic growth (Tin et al., 2014) [13].

Fake news concentration among medical students in Saudi has been perpetuated by identity theft that equates to intellectual property theft. In light of this, students are unaware of the verifiable information as publishers take advantage of the gullible student population to engage in identity theft. For instance, a journal may align its allegiance to a particular publisher. Understandably, fake news concentration among medical students has further been driven plagiarism 
of content. This implies that authors of journal articles refrain from scholarly research in the compilation of their data, therefore, use other authors' data without a clear representation of borrowed information leading to plagiarism. In medical science, for example, a significant portion of documented data is achieved through strenuous experiences from data compilation, analysis and findings to sustain an argument. However, the inclusion of plagiarized content kills off the quest for verifiable and factual information.

\section{Library Literacy Programs}

\section{Critical Thinking}

Critical thinking as a library literacy initiative helps information enthusiasts engage in reasoning prior to making conclusions in academia. For instance, a student reading academic material in a library should ensure that he/she engaged in critical thinking as fake news at times are identifiable at first sight. Critical thinking refers to a situation whereby the students identify a problem, explore a variety of solutions, determine best solutions, plan and implement solutions as well as conduct an evaluation of the results. Through critical thinking sessions offered to medical students at the university as basic course units, students are given an opportunity to upscale their literacy levels. For example, critical thinking lessons help students understand where, when and how to use information gathered from diverse areas such as physical or virtual libraries.

There are three critical categories of library literacy programs namely; behaviorist approach, constructivist approach and relational approach. First and foremost, the behaviorist approach is structured in a way that learners have certain characteristics of being information literate. In a behaviorist approach, learners are given an opportunity to define the task ahead, create information seeking strategies, locate and access information and use the information to synthesize results.

In a library setting, students are equipped with the best skills to ensure that they access the relevant information such that the intended results are achieved. This implies that students using electronic catalogs to search for information must first pen down the task to limit the provision of excessive information. The scaling down of information as propagated by the behaviorist approach ensures that learners are left with the finest research data for use in their studies.

Acknowledging its roots to the educational theories of Bruner, Vygotsky, and Kelly, the constructivist approach helps students adopt the unique model of information processing. In light of this, students using the constructivist approach in the literacy programs get an opportunity to engage in critical thinking where they have pre-set solutions to the topics they are conducting research on. For example, a learner who has prior information to a certain topic is unlikely to fall prey to fake news precisely because the information gathered earlier helps in the research process.

The constructivist model acknowledges a six-stage process namely; introduc- 
tion, selection, exploration, formulation and collection, and presentation. In a library setting, for instance, the introductory part represents a situation where a learner is guided by information processing. A librarian, in this context, charts with the learner to understand the feeling and composure prioritizing available information.

Fundamentally, it creates a perception that the learner engages in reflective searching. The relational approach to information literacy advocates learners' inclusion of perceptions and utilization of the same in understanding concepts. In a medical student perspective, to better understand a certain topic, he/she must internalize personal perceptions and integrate with research findings to come up with conclusive results.

Cognizant of their gateway roles to knowledge and culture, libraries are significantly resourced with books; electronic and print thus creating an opportunity for numerous persons to identify access and use any resource required. However, due to the influx of fake news occasioned by among others the overutilization of the Internet as an alternative information literacy avenue, has contributed negatively to the impeccable role of libraries in transforming an illiterate society to a literate one. Public libraries easy and affordable access, for example, helps in helping the students as well as the general population gain knowledge that is important for social, economic and political development primary because it broadens critical thinking.

Library literacy programs are important in modern day schooling environment cognizant of among others students' diversity and structured unique college academic procedures. In essence, the library literacy programs help student acclimatize with the academic environment associated with the courses they are pursuing. Fundamentally, in the interest of time, the students that undertake library literacy programs are likely to spend less time at the library locating the best literature as well as amplify their research with the best content.

Ina diverse school environment, enrolling in library literacy programs helps students adapt to the language of instruction at the institution. For example, a student enrolled in a Saudi Arabian university but is not conversant to the Arabian language may be helped in interpreting content written in Arabian thus simplifying his/her academic journal. This is evident in a multicultural society where information dissemination varies from one culture to another. Library literacy programs help learners align their priorities

\section{Challenges of Libraries in Fighting Fake News Infrastructural Stagnation}

With the increased need to digitize libraries as exemplified by the Saudi Digital Library to tap into emerging student population, lack of proper infrastructural development has hindered the growth of libraries. Interestingly, management of information systems requires significant government allocation of resources but due to reduced energies to achieve this, the growth of libraries faces imminent 
problems. Also, lack of proper infrastructural developments to equip libraries with professional and quality content is paving the way for fake news propagators to express their actions. For example, if a student lacks the precise literature to use in his/her studies and with the proximity of the Internet, he/she is likely to fall prey to the fake news thus jeopardizing academic excellence.

Fundamentally, inadequate room for expansion coupled with stringent publishing policies is also some of the challenges that libraries face in the pursuit for knowledge empowerment. This is because the stringent publishing policies scare away scholars against publishing their work considerably limiting the available content in the digital and physical databases. For example, when a digital library has inadequate academic literature to be used by students, redundancy is inevitable.

Additionally, libraries are faced with inadequate human expertise and modern information management systems to detect fake news and consolidate only viable and factual information. From this perspective, librarians in digital and physical libraries are inadequately capacitated to differentiate between factual and fake news. Ideally, the explosion of Internet-sites to supplement available information sources has further affected the growth of the knowledge-based society. For example, using the smart phone, a learner is likely to access fake news on social media pages faster compared to library material thus posing a major threat to the sustainability of the libraries. With the emergence of the Saudi Digital Library, institutions in the Arabic nation, for example, are strategically positioned to weed out pockets of fake news that may penetrate their academic systems (Bazrafkan et al., 2017) [15]. The available human resource in libraries is ill-equipped cognitively to address and merging demand for library services. This implies that the available librarians are incapable of dealing with the increasing need for library literacy across diverse academic fields. For example, a librarian is unable to assist a medical student search for literature and political science thus affecting the performance index. Libraries, moreover, are faced with the challenge of digitization. This is because the digitization process consumes a significant portion of resources namely money, physical infrastructure, and human resource.

To this end, the libraries are caught up in the information technology growth thereby becoming redundant in a changing learning environment. For instance, due to an increase in open access journals, learners are nowadays obliged to use digital libraries as opposed to physical libraries thereby affecting the sustainability factor. Libraries are sustained primarily from access fees and grants, but with reduced patronage, sustainability in the face of emerging digital platforms becomes a herculean endeavor.

\section{Conclusions}

Libraries have continued to play a critical role in the submission of knowledge to its recipients' across the world ranging from students, faculty members to the 
general public. However, the increase in the information sources perpetuated by technology such as the Internet has extensively affected the free form of authentic and factual information to the public. As illustrated in this research paper, it is important for institutions of higher learning to scale up their competitive advantage in the provision of knowledge through among others' integration of digital libraries. The influx of fake news on online platforms aimed at compromising academic integrity. To this end, it is vital for learners to be equipped with the necessary information that helps them identify, choose and use factual information.

Precisely, library literacy programs also help learners engage in critical thinking. In this regard, the programs instill critical thinking skills in learners such that they can use only information that they are sure of factual backing. For example, critical thinking entails extensive reasoning whereby students search for facts in any story or literature before confirming its authenticity. Penetration of fake news has been compounded by an increase in social media sites as well as websites canvassing as factual information generation platforms. To curb the escalating cases of fake news in learning institutions as well as the general population, publishers must come up with policies to limit the publication of literature to their platforms without adequate analysis. Additionally, social networking sites that have in the recent past increased tremendously should initiate regulatory measures to combat the negative impacts of fake news to millions of persons across the world. Ideally, social media platforms, as well as mainstream media institutions, should undertake self-censorship initiatives to gag any information that may misrepresent professional facts. For instance, a journalistic piece that is aired without the facts but focuses on sensational news injures the collective goal of library literacy programs initiated by institutions of higher learning across the globe.

This research paper recommends the needs for institutions of learning to come up with library literacy programs at the basic level to the tertiary levels of education to encourage scholarly excellence. In conclusion, there is an emphatic need for adequate information security systems to be initiated to avert compromising intellectual property.

\section{Disclosure}

The authors declare that no funding was received for this paper.

\section{Conflicts of Interest}

The authors declare no conflicts of interest regarding the publication of this paper.

\section{References}

[1] Baro, E.E., Endouware, B.C. and Ubogu, J.O. (2011) Information Literacy among Medical Students in the College of Health Sciences in Niger Delta University, Nigeria. Program, 45, 107-120. https://doi.org/10.1108/00330331111107439

[2] Qureshi, S., Qureshi, M.F. and Qureshi, V.F. (2018) Fake Journals, Spurious Pub- 
lished Papers, and Bogus Impact Factors: Need for an Overhaul and Transparency for an Academic Perspective. International Journal of Medical Science and Innovative Research, 3, 105-111.

[3] Okuonghae, O. and Ogiamien, L.O. (2016) Information Literacy Skills as Correlates of Library User Satisfaction among Undergraduates in Nigeria. Information Impact: Journal of Information and Knowledge Management, 7, 46-55.

[4] Zervas, M., Stavrou, C. and Kounoudes, A. (2015) The Important Role of School Libraries in the Development of Students Information Literacy Skills. Paper presented at the 7 th International Conference on Qualitative and Quantitative Methods in Libraries, Paris.

[5] Courtney, I. (2018) In an Era of Fake News, Information Literacy Has a Role to Play in Journalism Education in Ireland. Irish Communication Review, 16, 19-33.

[6] Nichols, T. (2017) The Death of Expertise: The Campaign against Established Knowledge and Why It Matters. Oxford University Press, New York.

[7] Bin Abdulrahman, K., Harden, R. and Patrício, M. (2012) Medical Education in Saudi Arabia: An Exciting Journey. Medical Teacher, 34, S4-S5.

https://doi.org/10.3109/0142159X.2012.660509

[8] Alsuraihi, A.K., Almaqati, A.S., Abughanim, S.A. and Jastaniah, N.A. (2016) Use of Social Media in Education among Medical Students in Saudi Arabia. Korean Journal of Medical Education, 28, 343-354. https://doi.org/10.3946/kjme.2016.40

[9] Mandalia, S. and Parekh, S. (2017) Awareness and Utilization of Digital Library by Library Users of ARIBAS Colleges: A Study. The International Journal of Indian Psychology, 4, 77-87.

[10] Storie, D. and Campbell, S. (2012) Determining the Information Literacy Needs of a Medical and Dental Faculty. Journal of the Canadian Health Libraries Association, 33, 48-59. https://doi.org/10.5596/c2012-011

[11] Stein-Smith, K. (2017) Librarians, Information Literacy and Fake News: Helping Students to Tell the Difference between Alternative Facts and the Real News. Strategic Library, No. 37, 1-23.

[12] Tin, L., Blesic, I., Biljana, B., Bibić, L.I., Dragan, M. and Sakulski, D. (2014) Predatory and Fake Scientific Journals/Publishers-A Global Outbreak with Rising Trend: A Review. Geographica Pannonica, 18, 69-81. https://doi.org/10.5937/GeoPan1403069L

[13] Agosto, D. (2018) Information Literacy and Libraries in the Age of Fake News. ABC-CLIO, California.

[14] Neely-Sardon, A. and Tignor, M. (2018) Focus on the Facts: A News and Information Literacy Instructional Program. The Reference Librarian, 59, 108-121. https://doi.org/10.1080/02763877.2018.1468849

[15] Bazrafkan, L., Hayat, A., Abbasi, K., Bazrafkan, A., Rohalamini, A. and Fardid, M. (2017) Evaluation of Information Literacy Status among Medical Students at Shiraz University of Medical Sciences. Journal of Advances in Medical Education and Professionalism, 5, 42-48. 